\title{
On The Types of Ancient Mysterious Buddha and Their Identity Jiahua Jiang ${ }^{1, a}$ \\ ${ }^{1}$ Shenzhen Polytechnic. Xili Town, NanShan District, Shenzhen, P.R.China. \\ ajjh@szpt.edu.cn
}

\begin{abstract}
There is a special class of Buddha in the ancient Buddhist statues categories, and this kind of Buddha has the mysterious legend, namely Mysterious Buddha. There are three main types of Mysterious Buddha. The first type, in the early India and China, is the Mysterious Buddha, which often became the object of other copy statues, this kind of buddha is called Zhenrong Mysterious Buddha. The second, some are made in accordance with the Zhenrong Mysterious Buddha, these mimic Buddha in the eyes of people still have the same position as Zhenrong Mysterious Buddha, so this kind buddha is called Mimic Mysterious Buddha. In the third type, some statues do not appear like the Zhenrong Mysterious Buddha, and also contains mystery, which are called General Pray-responsive Buddha. The three types of Buddha statues accord with the same characteristic from the perspective of their making and format, and this paper will discuss these three kinds of Buddha in terms of their embodiment and identity.
\end{abstract}

Keywords: Mysterious Buddha; types; Identity; General Pray-responsive

\section{古代佛教瑞像的类型及其同一性研究}

\author{
蒋家华 ${ }^{1, a}$ \\ 1深圳职业技术学院. 西丽, 南山, 深圳, 中国. \\ ajjh@szpt.edu.cn
}

中文摘要. 在古代的佛教造像品类中, 有一 类特殊的佛像, 这类佛像具有神秘的传奇 性, 即所谓的瑞像。从瑞像造作的分类看, 主要有三种类型: 古代早期的瑞像无论是在 印度还是中土, 往往成为其它佛像摹造的粉 本, 这类瑞像称作真容瑞像; 其次是依照真 容瑞像摹造的佛像, 在信众心目中仍具有瑞 像同等的地位, 这种摹像同样涵具神秘性, 这类瑞像笔者称作拟瑞像。还有一种瑞像没 有明显呈现出与真容瑞像关系, 但仍然很灵
验, 这类佛像属于一般灵验瑞像。无论是那 种瑞像, 它们在被造作的过程中, 都必须遵 循真容瑞像的造作标准。因此，对于依照同 一尊瑞像作粉本而造作的其它所有佛像其 形制则均符合同一性特征。这种同一性成为 判定同一类瑞像的标志, 这种标志的基本特 征, 即是这些瑞像具有相同的形制。对于本 文而言，即是对真容瑞像、拟瑞像、一般灵 验瑞像的各自内涵与它们之间的同一性进 行的讨论和研究。 


\section{关键词：瑞像；类型；同一性；灵验性}

\section{1. 引言}

本文的讨论, 笔者主要从三个方面进行 展开。其一, 交待瑞像的具体内涵; 其次, 分别介绍真容瑞像、拟瑞像、一般灵验瑞像 的具体含义, 同时分期三者之间造像标准的 同一性。瑞像造作标准的同一性, 是指一尊 瑞像与摹造的瑞像具有“完全一致”的形制。 任何一尊瑞像的造作都有其参照的摹造对 象, 即所谓的粉本。通过一些造像文献资料 表明, 一些瑞像是直接与像主产生对应关 系。也就是说, 这些瑞像是直接依照像主本 人造作的, 这意味着这些瑞像具神圣的权威 性, 笔者把这类瑞像称作真容瑞像, 这类瑞 像具有独一无二性。还有一类瑞像同样具有 权威性, 它们是依照真容瑞像摹造的, 这类 瑞像称作拟瑞像 (简称拟像)。拟瑞像往往 是依据印度本土的瑞像造作, 文献中大多有 所记载, 数量上可以是单尊, 也可以是多尊, 每尊拟像都有相应参照粉本、母像 (真容瑞 像)。还有一类瑞像不太出名, 既没有交代 其原初的来历, 也没有说明其是否是拟像, 只是零星的出现在文献中, 体现其超然的神 异性, 这类瑞像称作一般灵验瑞像。这三类 瑞像由于跟像主的不同关系而进行区分, 笔 者之所以都冠以 “瑞像” 之名, 是为了与一 般的功德造像相区分, 下面分别论述。

\section{2. 瑞像的内涵}

关于瑞像的内涵, 佛教学者丁福保对瑞 像的定义为: “优填王始以梅檀作释迦佛之 形像, 瑞相圆满, 故名瑞像。《(大唐) 西 域记》(卷) 五曰: “城内故宫中有大精舍, 高六十余尺, 有刻檀佛像, 上悬石盖, 邬陀 衍那王之所作也。灵相间起, 神光时照, 诸 国君王恃力欲举, 虽多人数莫能转移。遂图 供养, 俱言得真, 语其源迹, 即此像也。初 如来成正觉已, 上升天宫为母说法, 三月不 还。其王思慕愿图形像, 乃请尊者没特迦罗 子, 以神通力接工人上天宫, 亲观妙相雕刻 梅檀, 如来自天宫还也。刻檀之像起迎世尊, 世尊慰曰: 教化劳耶? 开导末世。”[1]
从上面引文可以知道, 虽然丁福保没能给出 确切的瑞像定义, 但是可以从其定义里援引 的《大唐西域记》卷 5 中关于斿檀像的来历 过程可以归纳出瑞像的几个特点: 第一、斿 檀像与释迦的关系, 是 “亲观妙相雕刻梅 檀”，也就是说斿檀瑞像是对释迦佛真容的 直接描绘。第二、斿檀像的灵验与神显, 即 “刻檀之像起迎世尊”，说明斿檀像已具有 肉身的神异性, 能起迎世尊, 而不是一般寂 静不动的佛像。第三、从其制作的技术考察, 旃檀瑞像为立体雕刻 (塑) 像, 按今日之雕 刻术语而言为圆雕，既非浮雕，也非平面的 画像, 即 “城内故宫中有大精舍……有刻檀 佛像, 上悬石盖, 鸟陀衍那王之所作也”。 第四, 关于造像传说, 旃檀瑞像有神话的敷 衍, 特殊的来历, 如 “初如来成正觉已, 上 升天宫为母说法, 三月不还。其王思慕愿图 形像, 乃请尊者没特迦罗子, 以神通力接工 人上天宫, 亲观妙相雕刻梅檀, 如来自天宫 还也。刻檀之像起迎世尊。”该神话故事敷 衍非常完整, 交代了斿檀瑞像的具体来历。

相比较而言, 张弓先生对瑞像内涵的定 义更符合本文的研究, 在他的著作中, 也比 较全面的对瑞像内涵做了比较全面阐述: “中古时期有少量的佛教造像, 或因为来历 与形制不凡, 或因为名家所造, 或曾有异常 “灵应” , 释门与信众益之种种传说, 赋予 特殊的 “神性” ，得以长期传世，成为僧俗 朝野公认的 “瑞像”。早期瑞像神主是诸佛: 释迦牟尼、阿弥陀、弥勒等; 后有观音、文 殊、普贤菩萨、北方天王毗沙门、罗汉迦毗 摩罗等。瑞像大都可以移动。瑞像制成质料 有木 (含旃檀) 、石、玉、铜、金、铜胎馏 （镏）金、铁、夹咜等; 织成、结珠、绣、 画之像。由于神话传说附益在这些瑞像上, 使之具有特殊的像教文化内涵, 因而成为佛 教造像中更具神圣色彩的特殊品类。有的 “瑞像” 不仅是僧俗信众的一般膜拜对象, 而且是释门与皇家传袭供奉的圣物。瑞像崇 拜的出现是中古时期佛神信仰深化的表征 之一。”[2]张弓对瑞像内涵的描述应该是 比较丰富的, 但是失于宽泛。归纳为如下几 点: 不凡的来历与形制、名家所造、灵应 (即 神显)、像主的多样化（有佛、菩萨、罗汉、 护法神）、质料的多样性（如木、石、玉、 
铜、夹咜等）、神话敷衍、信众崇拜、释门 与皇家传袭供奉的圣物、中古时期佛神信仰 深化的表征等等特征。应该说, 这些瑞像的 内涵基本上是符合笔者在本文研究的瑞像 特征。

实际上, 对于瑞像内涵的认识还有几位 重要的学者, 如巫鸿认为瑞像是神祇本身; 日本佛教美术学者认为瑞像具有预言和肉 身的功能; 而段玉明先生则认为瑞像实际上 是佛主等尊主的替身, 可以替代佛主传播佛 法。而张光达、荣新江二位学者在他们的论 文中则认为瑞像的特征仅表现为它们神显 的特定瞬间。以上所有关于瑞像的论述鉴于 篇幅的关系, 文献中的诸多瑞像特征与本文 讨论的瑞像类型没有太大的关系, 故而对其 论述所在的文献不作例举, 特此说明。

综合以上多为学者对瑞像的认识, 笔者 认为瑞像是瑞像是对像主真容的如实摹写, 瑞相圆满，具有固定的形制，有着肉身化、 神异化的特性, 能替代像主传播佛法、护持 佛法等独尊雕塑佛像, 他们往往被敷演以各 种非凡的传说, 且具备持续神显的能力, 瑞 像已经脱离了 “偶” 的范畴, 成为信众心目 中的超能神祇。[3]该定义的特点在于, 明 确了瑞像与像主真容的关系, 以及强调了瑞 像的肉身化、神异化特性。

\section{3. 瑞像的类型与同一性}

在本节的讨论中, 笔者把瑞像分作真容 瑞像、拟瑞像、一般灵验瑞像进行讨论。在 分析过程中, 总结这些瑞像之间有着共同的 联系, 并顺带交待作为一般功德供养的佛像 与瑞像的关系。

\section{1 真容瑞像}

通常来讲, 除摹造的瑞像外, 其余的瑞 像都是真容瑞像。关于 “真容”一词, 主要 是指瑞像主真身, 如在世释迦、弥勒、观音 真容等。文献中对 “真容”一词的应用得较 多, 如《高僧传》卷 13 云: “昔忧填初刻 梅檀, 波斯始铸金质, 皆现写真容工图妙 相。”[4]这里的真容就是指释迦, 也是指 优填王和波斯匿王是直接参照释迦真容而 图写妙相的。另一方面, “真容”一词还暗 示 “肉身” 的存在。在一些关于造像的传说
文献中, 会刻意敷演瑞像是如何依照像主真 身 (真容) 标准定身打造, 使得该瑞像获得 与像主无异神圣性。任何对像主真身造作不 标准的造像, 都将不在瑞像之列, 成为瑞像 中的 “噟品”。为了理解瑞像的 “真容” 性, 《佛祖历代通载》卷 22 可以做出很好的解 释：“八年辛卯, 思报母恩, 遂升㔹利天为 母说法。优阗王自以久失瞻仰, 欲见无从, 乃刻斿檀为像。目胖连虑有缺陃谬, 躬以神 力, 摄三十二匠升切利天, 谛观相好三返, 乃得其真。既成, 国王臣民奉之犹真佛焉。 及佛自㔹利天复至人间, 王率臣庶同往迎 佛, 此像腾步空中向佛稽首。佛为摩顶授记 曰: “我灭度千年之后, 汝从震旦广利人天。” [5] 上面文献是对优填王造像众多传说版本 中的其中一个。引文中说优阗 (填) 王为刻 旃檀佛像, 委托佛陀的弟子目胖连使用神通 摄三十二匠（应释迦三十二相, 寓意一匠图 一相) 升切利天谛观释迦真容, 甚至三返切 利天方成（“乃得其真”) 。至于目犍连以 三十二匠三返切利天谛观释迦的原因, 是因 为目胖连担心斿檀造像 “有缺陃谬”。可见, 该尊斿檀瑞像是完全按释迦的标准仪相进 行造作, 为斿檀瑞像获得 “肉身” 神性, 接 替释迦传法, 制造依据, 并在信众中形成权 威。

这类真容瑞像除了上面例举的优填王 造斿檀瑞像外, 其他著名的真容瑞像还有弥 勒造金刚座真容瑞像（菩提瑞像）、番禾瑞 像等等。这些真容瑞像在其造像传说中无不 强调瑞像是如何直接根据像主真身图写, 以 体现该瑞像的权威性质。这些瑞像一旦建 成, 往往受到众多信徒的顶礼膜拜。需要指 出的是, 如前文所述, 真容瑞像在中土的一 些文献中, 还有不同的称谓。如道宣在其著 作中称真容瑞像为 “本像” , 道宣《律相感 通传》卷1云: “又问: “荆州前大明寺梅 檀像者, 云是优填王所造, 依《传》从彼模 来至梁, 今京师又有, 何者是本? ” 答云: “大明是其本像……” ” (略) 即今兴善寺 像是也，亦甚灵异，本像在荆州。”[6]

上面引文中看, 荆州前大明寺的斿檀像 被认为是本像, 而京师的斿檀像是仿造大明 寺斿檀像摹写的。因此, 在道宣看来, 荆州 大明寺斿檀像由于是优填王所造, 故称其为 
“本像”, 主要是为了与其它摹造的佛像进 行区分。在笔者看来, 对于真容瑞像以 “本 像” 为称谓, 不够确实, 本像在本质意义上 其实是摹像参照的 “第一粉本” 而已。而作 为 “粉本” 的本像, 也有可能是摹造的, 具 体研究中需要细加甄别。

需要指出的是, 每尊真容瑞像是独一无 二的, 且具有唯一性。诸如弥勒造金刚座上 尊像 (菩提瑞像) 仅一尊, 唐时存放在印度 佛教圣地菩提伽耶, 任何其它地方的金刚座 真容菩提瑞像都将是摹本, 其权威性也意味 着相对降低。

\section{2 拟瑞像}

拟瑞像是指以真容瑞像为标准粉本进 行如实摹写与造作的瑞像, 这类瑞像同样具 有相对的权威性, 特别是受到中土帝王、僧 徒、以及普通信众的青睐与追捧。关于 “拟 瑞像”一词的称谓, 来自于玄獎《大唐西域 记》卷12: “拟摩揭陀国前正觉山龙窟影像 金佛像一躯, 通光座高三尺三寸; 拟婆罗痕 斯国鹿野苑初转法轮像刻檀佛像一躯, 通光 座高尺有五寸; 拟憍赏弥国出爱王思慕如来 刻檀写真像刻檀佛像一躯, 通光座高二尺九 寸; 拟劫比他国如来自天宫降履宝阶像银佛 像一躯, 通光座高四尺; 拟摩揭陀国鹑峯山 说《法花》等经像金佛像一躯, 通光座高三 尺五寸; 拟那揭罗曷国伏毒龙所留影像刻檀 佛像一躯, 通光座高尺有三寸; 拟吠舍厘国 巡城行化像。”[7]

上面引文的内容是玄裝西行回国所带 回的佛像清单。在文字的措辞上, 每一尊佛 像前面都有一 “拟” 字, 是为了与其本像作 区分, 也就是说所有这些拟像的本像都可能 在印度有其粉本或摹造的原型。因此, 笔者 把“拟瑞像”视作是对真容瑞像的标准摹造。 由于真容瑞像的独一无二性、唯一性, 从整 体上看, 这类瑞像的数量极其有限。有时候 信徒们为了瞻仰真容, 不可能人人都能去真 容瑞像的安置处朝圣。为了解决这个问题, 一些技法高超的造像匠人便依照真容瑞像 进行摹造, 故而成为拟瑞像（简称拟像）, 然后根据各地的信仰需要进行传播。由于某 些真容瑞像特殊的灵验性, 往往吸引众多信 徒对真容瑞像竞相摹造(所谓“道俗竞摸”)。 他们在造摹造过程中, 由于摹写的要求极
高, 通常很难成功。下面的例子说明了对瑞 像的摹造的困难: “其像（金刚座真容菩提 瑞像) 自弥勒造成已来, 一切道俗规模图写, 圣变难定, 未有写得。” [8]可见拟瑞像造 作的困难, 非技术熟练的造像匠人不可 (后 由匠人宋法智摹写成功, 带回中土)。同时, 需要指出的是, 拟瑞像造作的参照对象, 一 般都是选择那些著名的真容瑞像进行摹造, 而一些不太知名的瑞像, 则很少引起信众们 的关注。

与真容瑞像是由无漏之身 “神人” 所造 不同的是, 拟瑞像一般是由人工造作完成。 因此, 就造作主体方面体现了真容瑞像与拟 瑞像的本质差别, 同时折射出二者在神圣性 与灵验力方面的宗教神圣性差别。尽管如 此, 无论在古代印度还是中土, 信徒们对这 些拟瑞像仍然是当作神圣的真容瑞像进行 供奉, 在他们心目中, 这些拟瑞像与真容瑞 像是可以同等的, 无有二至。

\section{3 一般灵验瑞像}

如前所述, 一般灵验瑞像往往是出现在 中土的一些文人编撰的志怪小说、佛教感应 故事录中。这部分瑞像大多没有太清晰的来 历, 其造作也没有介绍其明显的参照对象, 也不是僧俗信众摹造的对象, 但仍然充满灵 验性。由于这类瑞像数量相对较多, 在后面 章节的论述中将有详细介绍, 此处不再作出 例举。

以上三类瑞像, 是根据瑞像造作的参照 对象作出的分类, 但对于信众而言, 他们不 会去区分这些瑞像是真容瑞像、亦或拟像, 甚至还是一般的功德造像之间的区别, 正如 段玉明所认为的那样, “对信众而言, 区别 真身与替身既不可能也无必要。信众需要这 个替身 (他们甚至把一些功德造像也视作真 身。笔者注）, 用以激起、唤醒他们对那不 可临现的真身的情感。而且, 通过圣显的介 入, 真身与替身的确也已无法分辨。自道宣 始, 中国僧人一直非常重视造像的 “感通”, 说明他们相信这些造像具有灵性一一可以 与僧众和信众在神秘的层面往复交流，而绝 不只是艺术品似的泥塑木雕。” [9]也就是 说, 普通的信众他们对瑞像的崇拜是不需要 分辨瑞像的类分属性, 在他们眼中, 这些造 像都等同是释迦等神主的亲自到场。这就解 
释了为什么大量的功德像为什么会以瑞像 的名义进行雕業, 在有些造像记中还冠以 “真容” 等词汇。对于真容性而言, 即这些 佛像与释迦在生时的容貌没有本质差别, 另 一方面, 信众把佛像作为真容、释迦生身看 待, 实际上还是为了施加对释迦的缅怀。因 此, 所有这些瑞像, 在古代普遍受到大量信 众的膜拜, 这是由他们的信仰和宗教感情所 决定的。

通过以上关于瑞像造作参照不同类型 的分析, 笔者把真容瑞像、拟瑞像、功德 (以 瑞像为题材) 造像之间的关系, 得出下面的 程式（图1）：

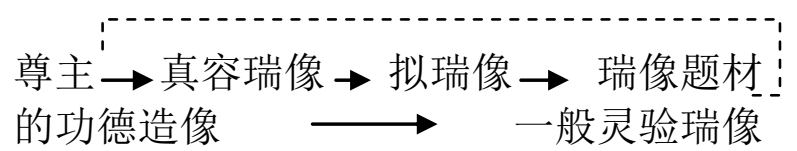

图1 肉身尊主与瑞像、功德造像的关系图式

上面循环图中箭头表示前者是后者的 参照对像, 实线表示前后造像之间的逻辑参 照关系, 只有一般灵验瑞像是直接与尊主相 关。从上图我们也可以看出从瑞像到以瑞像 为题材的功德造像, 出现了瑞像崇拜泛化的 趋势, 瑞像权威性或神圣性的逐渐降低, 而 一般灵验瑞像则一直出现在佛教感应录、以 及一些文人的志怪、笔记小说中。从上面的 循环图式还可以看出, 从尊主到一般灵验瑞 像, 他们之间实际上共同分享着尊主的权威 性、灵验性, 使得各种类型的瑞像之间保持 着同一性。

\section{4. 结束语}

本文从藏内文献的角度对早期佛教特 殊的造像瑞像的内涵和类型进行了基本的 讨论。一方面, 早期的这类瑞像具有特殊的 神秘性, 以及相应的神话传说。另一方面, 在佛教造像艺术史的发展过程中, 我们除了 依据现存地上文物和考古文物来研究佛像 艺术的形态, 同时也可以从纯文献的角度考 察早期佛教艺术发展受到的相关影响。这种 影响, 即是对于佛像的匠作而言, 这些同一 性的标准对他们具有特殊性的约束力, 即不 得妄造佛像。另一方面, 对于瑞像品类之间 造作同一性的遵循, 保持了前后造像风格的 传承性, 这无疑具有积极的意义。但根据现
存考古资料, 佛像的造作风格受到的影响实 际上更多的来自本地民族的审美习惯, 在本 文中, 这已经不是该文讨论的范围了。因此 对于瑞像而言, 更多承载的是其宗教性内 涵, 而非艺术性, 这是通过本文研究得出的 结论。

\section{致谢}

该文为国家社科基金重大项目 “多卷本 《中国寺观文化史》”(项目编号: 13\&ZD079) 的阶段性成果。并对该项目团队对本文的建 议和支持表示诚挚的感谢。

\section{References}

[1] Ding Fubao, Dictionary of Buddhism, Cultural Relic Press, pp.1199, 1984.

[2] Zhang Gong, The Culture History Of Buddhist Temple During Han and Tang Dynasties, China Social Science Press, pp.662, 1997.

[3] Jiang Jiahua, Research on Generation of the Ancient Indian Buddhist Pratima, Social Sciences in Yunnan, vol.6, pp.138, 2013.

[4] Hui Jiao, The Biography of Eminent Monks, vol.13, CBETA, T50, no. 2059, pp.413, a10-11.

[5] Chang Nian, The Buddha's History, vol.22, CBETA, T49, no. 2036, pp.730, c22-23.

[6] Dao Xuan, The Pray-responsive of Law, vol.1, CBETA, T45, no. 1898, pp.877, b25-c4。

[7] Xuan Zang, Datang Westernregions, Vol.8, CBETA, T51, no. 2087, pp.946, c3-15.

[8] Dao Shi, Fayuan Zhulin, vol.29, CBETA, T53, no. 2122, pp.503, a7-12.

[9] Duan Yuming, Xiangguo Temple, Bashu Press, pp.134, 2004 